\title{
Mapeamento de iniciativas estrangeiras em língua inglesa que fomentam a entrada de mulheres na Computação
}

\author{
Maria Augusta S. N. Nunes ${ }^{1}$, Carolina S. Louzada ${ }^{1}$, Edilayne M. Salgueiro ${ }^{1}$, \\ Beatriz T. Andrade1, Patrícia S. de Lima ${ }^{2}$ Raquel M. C. T. Figueiredo ${ }^{1}$ \\ ${ }_{1}$ DCOMP/PROCC- Universidade Federal de Sergipe/UFS - São Cristóvão- SE \\ ${ }_{2}$ Escola Estadual Atheneu Sergipense -Aracaju - SE \\ gutanunes@gmail.com, carolina.louzada@hotmail.com, edilayne@ufs.br, \\ beatriz@ufs.br, paslima@globo.com, raquelmctf@gmail.com
}

\begin{abstract}
This paper provides an overview of foreing initiatives based on english language that promote women into the computing area around the world. The methodology used was based on Systematic Mapping. As a conclusion we found that the foreing initiatives of women in the computing area based on english language is quite representative wordwide that could encourage the migration of women in computing.
\end{abstract}

Resumo. Esse artigo tem como objetivo obter um panorama das iniciativas estrangeiras baseadas em língua inglesa que fomentam a entrada de mulheres da área da Computação no mundo. A metodologia utilizada foi inspirada no processo de Mapeamento Sistemático. Concluiu-se que há, atualmente, uma significativa quantidade de iniciativas estrangeiras baseadas em língua inglesa distribuídas mundialmente que fomentam a entrada de mulheres na área da Computação.

\section{Introdução}

$\mathrm{Na}$ Computação, as dificuldades e a falta de incentivo refletem no baixo número de mulheres ingressantes em cursos dessa área. O baixo percentual de mulheres nessa área não é só preocupante com relação às tentativas de minimizar as desigualdades dos gêneros, mas também preocupa o mercado de Tecnologia da Informação (TI) atual. Diante desse contexto, há uma crescente preocupação em criar estratégias de incentivo que visam diminuir essa baixa representatividade das mulheres na Computação [Louzada e outros 2014]. Existem algumas estratégias e ações, tais como a da Universidade de Stanford que disponibiliza um curso introdutório de Computação que foi adaptado para atender um leigo, além dos tradicionais graduandos da área; nesse curso foram apresentadas personalidades importantes na área de Computação incluindo também mulheres de sucesso. Outras diversas universidades tem criado ações, tais como minicursos ou oficinas para alunos de ensino médio, onde são apresentados os conceitos de programação e lógica de programação [Gomes e outros 2014; BlackGirlCode 2015]; ações, também, apresentadas em forma de concursos que estimulam o empreendedorismo desde o ensino médio, para fomento ao gênero [Technovation 2015]. Durante essas ações tem se percebido que as meninas, geralmente, não conhecem o perfil do profissional de TI e, consequentemente, não vislumbram as oportunidades de trabalho na área, esse é um dos fatores que pode determinar a falta de interesse delas nos cursos da área de Computação. Observa-se, também, que a formação de grupos de 
suporte às mulheres nas universidades auxilia na retenção dessas mulheres nos cursos de Computação [Beck 2007], demonstrando que as medidas adotadas nas universidades podem ser um importante estímulo para manter o interesse das mulheres no mercado de TI. O objetivo deste artigo, então é buscar e quantificar iniciativas estrangeiras ${ }^{1}$ baseadas em língua inglesa de fomento ao gênero para a área da Computação. Nesse artigo utilizou-se o método de Mapeamento Sistemático de URLs relacionados à inclusão de mulheres na área de Computação e afins no contexto estrangeiro usando a língua inglesa como base. Esse trabalho está organizado da seguinte forma: na Seção 2 descreve-se a metodologia; na Seção 3 realiza-se uma análise dos dados encontrados e na Seção 4 descreve-se as considerações finais.

\section{Metodologia}

A busca por iniciativas que estimulem o ingresso de mulheres na área da Computação é um trabalho amplo. Não há uma metodologia específica para verificar e garantir que todas as iniciativas estrangeiras baseadas em língua inglesa existentes estejam todas incluídas na pesquisa. Foi utilizada uma adaptação do método do Mapeamento Sistemático (MS), proposto por Petersen e outros (2008) e Kitchenham (2007). De acordo com ambos os autores, o MS é uma metodologia que fornece uma visão geral do que se quer mapear por meio da quantificação de evidências. A partir dessa quantificação é possível verificar e interpretar os dados encontrados e, dessa forma, encontrar tendências que são essenciais para a formação de um panorama do tema abordado. As etapas executadas foram divididas da seguinte forma: (i) Definição das questões de pesquisa; (ii) Busca de iniciativas, projetos e organizações; (iii) Seleção de iniciativas, projetos e organizações relevantes; (iv) Extração e classificação de dados; (v) Análise dos dados. Os dados foram coletados em Agosto de 2014.

\subsection{Questões de pesquisa e busca de projetos e iniciativas}

$\mathrm{Na}$ primeira etapa do método, questões foram definidas de forma a limitar os dados procurados. As questões de pesquisa proposta foram: 1. Quais e quantas são as iniciativas estrangeiras baseadas em língua inglesa com base nas informações encontradas na web? 2. Quando essas iniciativas foram criadas (ano de fundação)? 3.Em quais países essas iniciativas são aplicadas? Na segunda etapa, utilizaram-se strings de busca para pesquisar essas iniciativas estrangeiras baseadas em língua inglesa por meio de URLs. Como não há uma base de dados científica para os tipos de dados procurados, tal como SCOPUS, IEEE, ACM, SPRINGER, como recomenda a metodologia de mapeamento sistemático, foi realizada uma adaptação metodológica e, então, recorreu-se a uma base de dados da Internet. Foi utilizado o motor de busca Google, que retorna os resultados mais acessados e populares de acordo com as strings pesquisadas. O motor de busca, retornou então, uma quantidade significativa de resultados, mais de 600 milhões. De forma a limitá-los, estipulou-se que os dados seriam procurados nas dez primeiras páginas encontradas, visando criar um panorama inicial para o mapeamento. Foram revisadas 100 iniciativas, na língua inglesa, com os mesmos termos:((TITLE: woman OR TITLE: women) AND (TITLE: computer)); ((TITLE: woman OR

\footnotetext{
${ }^{1}$ A palavra « estrangeira » refere-se a « fora do Brasil ». Nesse artigo não usou-se o termo « internacional «pois objetivou-se mapear as iniciativas desenvolvidas fora do Brasil, fossem elas internacionais ou não. Exemplificando, conforme a visão dos autores, uma iniciativa brasileira, pode ser considerada internacional, quando ela atinge um público estrangeiro, uma forma de atingir esse público é a página web da inciativa estar desenvolvida, também, em uma língua estrangeira, como o inglês, por exemplo.
} 
TITLE: women) AND (TITLE: computing)); ((TITLE: woman OR TITLE: women) AND (TITLE:information technology)).

\subsection{Critérios de inclusão e exclusão e extração de dados}

Tendo a relação dos URLs encontrados na pesquisa, utilizaram-se critérios de inclusão e exclusão para encontrar os sites com dados relevantes para a pesquisa. Os sites incluídos foram aqueles, na língua inglesa, criados pelas iniciativas que apresentam propostas estrangeiras que incentivem especificamente mulheres no campo da Computação ou diminuam sua evasão. Já os excluídos, classificados neste artigo como não relevantes, não estão na língua inglesa, não são brasileiros e não apresentam propostas de incentivo às mulheres na Computação ou somente mencionam as iniciativas e projetos, como no caso de sites jornalísticos. A partir desses critérios o número de sites foi reduzido. Dos cem sites encontrados no total, cinquenta e nove foram de iniciativas estrangeiras baseadas em língua inglesa, sendo elas:Women in Technology (Austrália), IEEE Women in Engineering, NCWIT, Women in Technology(Reino Unido), Associação Profissional WITI, WIT Education Foundation, Technovation Challenge, Blog ACM-W COUNCIL WOMEN IN COMPUTING NEWS, Railsgirls, Girls who code, she + +, pyladies, Women techmakers, ACM-W, The Center for Women In Technology( CWIT), Debian, Genderchangers, Women into Science and Engineering, IWT, Fabfems, National Girls Collaborative Project, The Computer Research Association's Committee on the Status of Women in Computing Research, Women in Engineering and Computing Career Day, Women in Computer Science, Arabwic, Maryland Center for Women in Computing, Association for Women in Computing, Valentina project, Jinnah University For Women, Anitaborg, College of Computing and Information Women in Technology, Young Women in Computing, Ada initiative, woman@CC, Black girls code, Women in Computer Science at North Carolina State University, NSF ADVANCE Program, CloudNOW, BinaryGirl, EngineerGirl, Institute for Women in Trades, Technology \& Science's,Women in games international, Girlstart, DIYgirls, Her ideas in motion, Girls in Tech, Duchess-france, Girls learning code, Linuxchix, Projeto Ubuntu Women, Women in Computer Science in Boston University, Webgrrls, Devfest, Women2, Million women mentors, women in stem, Women who code, Digigirlz (Links disponíveis em: (http://200.17.141.213/ gutanunes/wit2016/apendiceEstrangeiro.pdf) Após a seleção dos sites que atenderam aos critérios foi possível extrair os dados necessários. Esses dados analisados ajudaram a responder às questões de pesquisa definidas. Os dados extraídos foram: o título da iniciativa, países de alcance ou ação e ano de fundação.

\section{Análise dos dados}

Muitas das iniciativas pesquisadas utilizam mais de um modo/fonte de fomento. Neste contexto, destacam-se as organizações criadas especificamente com o propósito de incentivar mulheres e universidades que desejavam inspirar e aumentar o número de mulheres em seus cursos de Computação. A análise desses dados é apresentada nas subseções seguintes.

\subsection{Catalogação, quantidades e tipos de iniciativas}

De acordo com a metodologia, o primeiro passo foi catalogar o título e as URLs das respectivas iniciativas estrangeiras baseadas em língua inglesa. Por meio da leitura das propostas contidas nos sites das iniciativas foi possível verificar os tipos de iniciativas pesquisadas. Ao final da leitura dessas iniciativas/propostas foram verificados os seguintes tipos de ações: workshops, palestras, fóruns, tutoria, cursos, conferências, consultoria, publicidade, agenciamento, competição, jogos de ensino, desenvolvimento de softwares, programas pedagógicos e blogs. O Gráfico 1 apresenta essas classificações 
obtidas dentre as diversas iniciativas estrangeiras baseadas em língua inglesa.

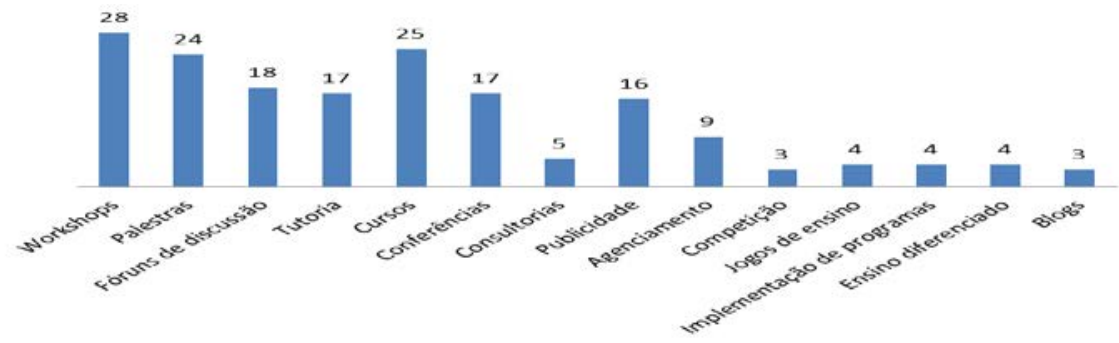

Gráfico 1. Relação entre quantidade e tipos de iniciativas estrangeiras

\subsection{Ano de fundação}

Com relação às iniciativas estrangeiras baseadas em língua inglesa verificou-se um grande contraste nos anos de fundação, como é visto no Gráfico 2. A iniciativa mais antiga encontrada na pesquisa foi fundada no ano de 1978. A criação de uma ou duas iniciativas por ano manteve-se até o ano de 2003, em que houve o lançamento de duas iniciativas estrangeiras baseadas em língua inglesa. A partir de 2004 a média de iniciativas aumentou e no ano de 2012 verificou-se o maior número de iniciativas criadas, com a criação de sete iniciativas. Em 2013 novamente esse número caiu e voltou a crescer em 2014. Devido à falta de informações nos sites não foi possível extrair a data de criação de quatro iniciativas. Contudo, essa falta de informação não prejudica o entendimento quanto ao crescimento de iniciativas de incentivo às mulheres na Computação, sejam públicas ou privadas.

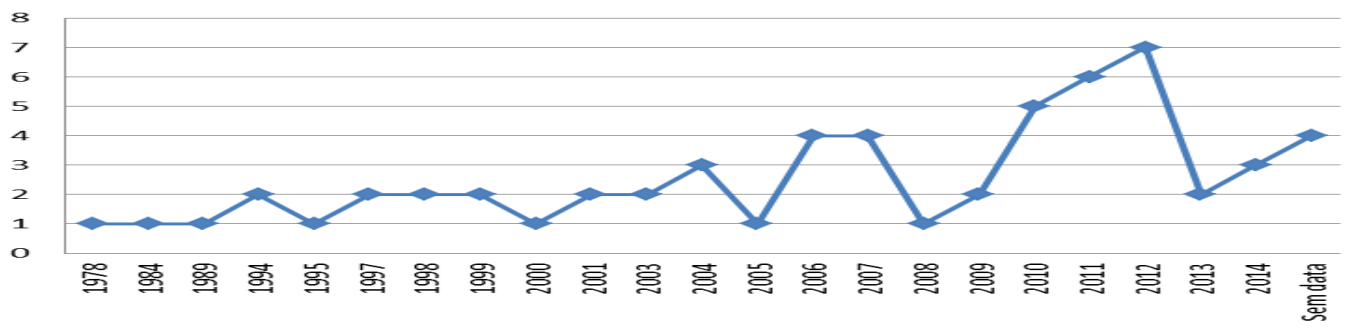

Gráfico 2. Ano de fundação das iniciativas

Em Louzada et. al (2014) foi encontrado no ano de 2002, o maior número de publicações científicas sobre o incentivo a entrada de mulheres na Computação. Comparando com o Gráfico 2, no mapeamento de iniciativas, verifica-se que dez anos depois, em 2012, foram criadas o maior índice numérico de estratégias de incentivo. A primeira publicação relacionada surgiu em 1975, no entanto observa-se que a primeira iniciativa foi fundada somente em 1978.

\subsection{Países de alcance}

A partir da leitura do Gráfico 3 verificou-se que $56 \%$ das iniciativas têm como foco os EUA, e em segundo lugar, as iniciativas de alcance internacional, com 32\%. As iniciativas de alcance internacional são aquelas que são criadas em determinado país e, também, atuam em outros países. O Canadá possui o menor percentual com relação ao número de iniciativas, ficando com $1 \%$. Também, com um baixo número de iniciativas, ocupando 2\% do total, tem-se a Austrália, França, Emirados Árabes e Paquistão. Essa relação entre países de alcance é importante para entender-se a forte conexão internacional entre diversos países que possuem a necessidade de incentivar as mulheres 
na Computação.
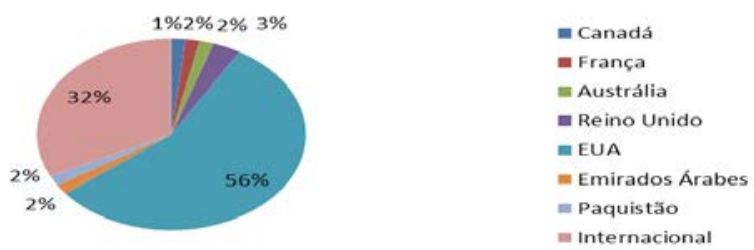

\section{Gráfico 3. Relação percentual dos países em que as iniciativas atuam}

\section{Considerações Finais}

Por meio do Mapeamento Sistemático, esse artigo teve como objetivo verificar as iniciativas estrangeiras baseadas em língua inglesa que fomentem a entrada de mulheres na Computação. Foram encontradas cinquenta e nove iniciativas estrangeiras baseadas em língua inglesa. A criação de iniciativas de alcance internacional mostra a preocupação latente em conseguir bons profissionais para o mercado de TI e diminuir a diferença percentual entre homens e mulheres na área. Os EUA são foco de $56 \%$ do total das iniciativas pesquisadas e isso pode mostrar um maior percentual na entrada de mulheres nos cursos de Computação com relação a outros países, mesmo considerando a língua base como o inglês. Muitos países que, também, possuem o inglês como primeira língua apresentam uma baixa representatividade, nesse mapeamento, em termos de iniciativa, tais como: Reino Unido com 3\%, Austrália com $2 \%$ e parte do Canadá (anglófona) com 1\%. Independente do país de origem das iniciativas, $36 \%$ destas iniciativas, em língua inglesa, tiveram um alcance internacional, ou seja, as iniciativas criadas em determinado país não se concentraram somente nesse mesmo país, mas buscaram atingir diversos outros países. Sugere-se para trabalhos futuros que se investigue outras línguas para que se tenha um panorama mundial concreto considerando a distribuição territorial das iniciativas nos dias atuais. É interessante também pesquisar sobre a relação dos países e o efeito causado pelas iniciativas na efetiva entrada de mulheres nos cursos de Computação de suas Universidades.

\section{Referências}

Beck, J. (2007). Forming a Women's Computer Science Support Group. In: ACM SIGCSE'07. EUA.

Gomes, W. F.; Louzada, C. S.; Nunes, M. A. S. N.; Salgueiro, E. M.; Andrade, B. T. (2014) Incentivando meninas do ensino médio à área de Ciência da Computação usando o Scratch como ferramenta. In: CBIE 2014 - 20 $0^{\circ}$ Workshop de Informática na Escola (WIE).

Kitchenham, B. (2007). Guidelines for performing Systematic Literature Reviews in Software Engineering, Technical Report. Keele University.UK.

Louzada, C. S.; Gomes, W. F.; Nunes, M. A. S. N.; Salgueiro, E. M.; Andrade, B. T.; Lima, P. S. (2014). Um mapeamento das publicações sobre o ingresso das mulheres na Computação. In: CLEI 2014 - VI Congresso da Mulher Latino-americana na Computação, Montevidéu.

Petersen, K.; Feldt, R.; Mujtaba, S.; Mattsson, M.(2008). Systematic mapping studies in software engineering, ACM. New York.

BlackGirlCode(2016). Disponível em: http://www.blackgirlscode.com/. Acesso 19/04/2016.

Technovation(2016). Disponível em:http://www.technovationchallenge.org/Acesso 19/04/2016. 